\title{
Estimating the Depth of Shower Maximum using the Surface Detectors of the Pierre Auger Observatory
}

\author{
Carlos José Todero Peixoto ${ }^{* a}$ for the Pierre Auger Collaboration ${ }^{b \dagger}$ \\ a Escola de Engenharia de Lorena, Universidade de São Paulo, São Paulo, Brazil \\ ${ }^{b}$ Observatorio Pierre Auger, Av. San Martín Norte 304, 5613 Malargüe, Argentina \\ E-mail: auger_spokespersonsefnal.gov \\ Full author list: http://www.auger.org/archive/authors_icrc_2019.html
}

\begin{abstract}
The Pierre Auger Collaboration has developed a method for estimating the depth of shower maximum $\left(X_{\max }\right)$ using the risetime of the signals recorded by the array of water-Cherenkov detectors. The average value of $X_{\max }$ is determined for energies above $3 \mathrm{EeV}$, using air showers with zenith angles up to $60^{\circ}$. The work described here considerably extends that reported previously with an increase in the total number of events by a factor 2.1. In total 125,005 events are used, of which 237 are above $50 \mathrm{EeV}$. The data set is approximately a factor 30 larger than what is available from fluorescence measurements for energies above $3 \mathrm{EeV}$. The results reinforce previous conclusions: the mean mass of high-energy cosmic rays begins to rise above $3 \mathrm{EeV}$ and extend that finding to higher energies with greater precision. Additionally, we find strong discrepancies when we make a comparison between observations and predictions from simulations with values which are obtained from measurements of the risetime of the recorded signals.
\end{abstract}

36th International Cosmic Ray Conference -ICRC2019-

July 24th - August 1st, 2019

Madison, WI, U.S.A.

\footnotetext{
${ }^{*}$ Speaker.

${ }^{\dagger}$ for collaboration list see PoS(ICRC2019)1177
} 


\section{Introduction}

Knowledge of the mass composition of cosmic rays would provide us with crucial information to discriminate between different classes of production, acceleration and propagation models, and to understand the origin of the observed flux suppression; it would allow us to search for a stream of protons at higher energies, thus enhancing the study of small-scale anisotropy. It could further improve previous studies of particle physics at $10 \mathrm{EeV}$ and extend them to energies as high as $\sqrt{s}=$ $200 \mathrm{TeV}$.

Using data from the Pierre Auger Observatory, several methods that enable mass discrimination with ground-based detectors have been developed. Of the several parameters studied, those that presented some relevance were $X_{\max }$ [1] (from measurements of the fluorescence detector, FD), $X_{\max }^{\mu}$ [2], Risetime Asymmetries [3] and the Delta Method [4,5], the latter three using the signals from the surface detector, SD. The $X_{\max }$ parameter has the best resolution for mass composition studies, but the FD only operates during nights without moonlight, only $13 \%$ of the operating time of the SD. With the Delta method, we can use the signals from the SD, which has a nearly $100 \%$ duty cycle, to calculate a parameter, $\Delta_{\mathrm{S}}$, and to convert this to an estimation of the depth of the shower maximum, $X_{\max }^{\text {Delta }}$.

This work is based on the Delta Method [4], which was originally used with SD data up to zenith angles $\sim 45^{\circ}$. We now extend the method to events up to $60^{\circ}$. This yields an increase of about $50 \%$ in the number of events, thus reducing the statistical uncertainty associated with masscomposition inferences. We have also extended the sample by including three additional years of data. The large data set from the $750 \mathrm{~m}$ array has not been updated.

\section{Data Selection}

This work uses a data sample from the $1500 \mathrm{~m}$ array collected from January 2004 to August 2018. We consider events with zenith angles below $60^{\circ}$, and with energies above $3 \mathrm{EeV}$ to guarantee full trigger efficiency.

We also impose conditions at the level of single water-cherenkov detectors (WCD): the total signal must be above 5 VEM, and the distance to the shower core must be in the interval [300, $1400] \mathrm{m}$ for the $1500 \mathrm{~m}$ array. For events with energies $\log _{10}(\mathrm{E} / \mathrm{eV})>19.8$, we extend the distance range up to $2000 \mathrm{~m}$.

After applying these selection criteria, the number of events used in what follows is 125,005 .

\section{The Delta Method}

The Delta Method is explained in Figure 1(Left). We characterize every event with a single parameter $\Delta_{S}$, which is a function of the risetimes ${ }^{1}$ recorded for this event. We have determined relationships (dubbed as benchmarks) that describe the risetimes as a function of the distance and zenith angle in a narrow energy range. Risetimes for each WCD are compared to the predicted benchmark value, in units of the accuracy with which risetimes are determined, giving a parameter

\footnotetext{
${ }^{1}$ This parameter is the time for the integrated signal to increase from $10 \%$ to $50 \%$ of the final magnitude of the integrated signal.
} 
$\Delta_{\mathrm{i}} . \Delta_{\mathrm{S}}$ is the average of the $\Delta_{\mathrm{i}}$ values over the N selected WCDs. An example of the dependence of the risetime with the distance to the core is shown in Figure 1(Right).

For each PMT in a detector, there are two Flash Analog-to-Digital Converter (FADC) channels: a high-gain and a low-gain channel (used when the high-gain (HG) channel has saturated). Fits to the data are made using the function

$$
t_{1 / 2}^{\text {low-gain }}=40 \mathrm{~ns}+\sqrt{A(\theta)^{2}+B(\theta) \cdot r^{2}}-A(\theta),
$$

where $A[\mathrm{~ns}]$ and $B\left[\mathrm{~ns}^{2} / \mathrm{m}^{2}\right]$ are free parameters. With $A$ and $B$ known, a fit to the points from the HG channel is made (shown by grey points in Figure 1(Right)) as

$$
t_{1 / 2}^{\text {high-gain }}=40 \mathrm{~ns}+N(\theta) \cdot\left(\sqrt{A(\theta)^{2}+B(\theta) \cdot r^{2}}-A(\theta)\right)
$$

where $N(\theta)$ is a free parameter. In this way, the small differences between the measurements with the two channels are dealt with.

These three parameters are parameterized as a function of $\sec \theta$ in the narrow benchmark energy bin. The energy bin used is $\log _{10}(\mathrm{E} / \mathrm{eV})=19.1-19.2$.
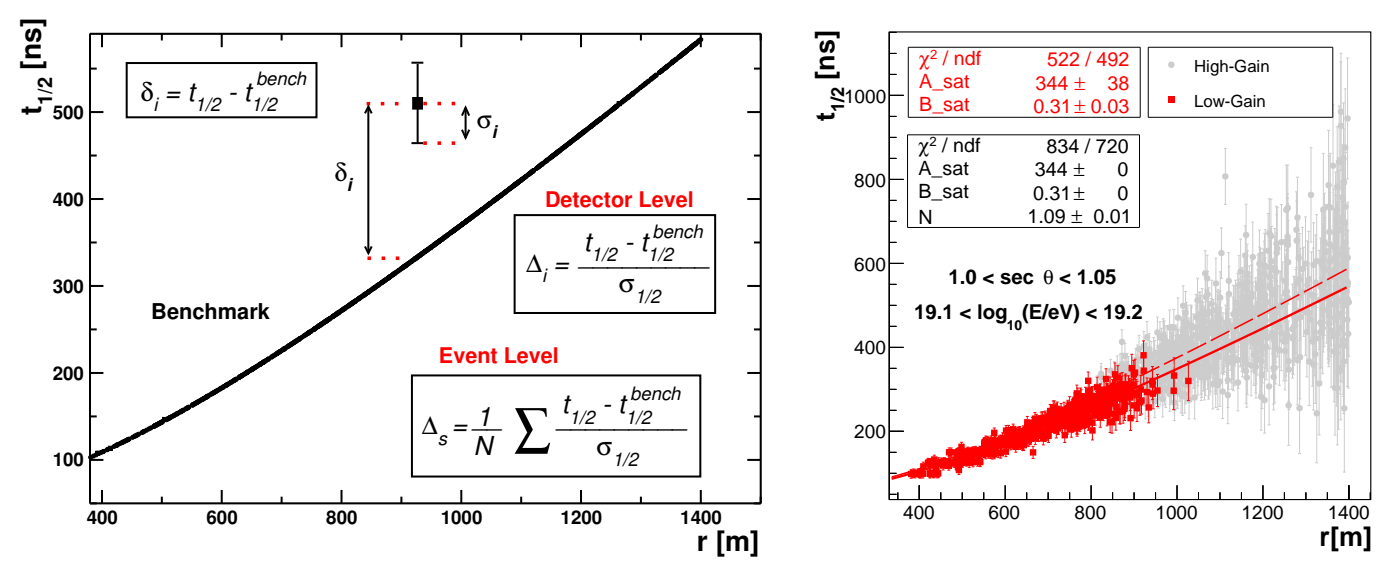

Figure 1: (Left) Description of the Delta Method. (Right) Risetime measurements as a function of the distance to the core for data from the $1500 \mathrm{~m}$ array in the energy interval $19.1<\log _{10}(\mathrm{E} / \mathrm{eV})<$ 19.2 .

The Delta Method procedures are fully described in $[4,5]$.

\section{Extending to angles up to 60 degrees}

Once the functional forms of $A(\theta), B(\theta)$ and $N(\theta)$ are known, the mean value of the $\Delta_{\mathrm{s}}$ distribution must be, by definition, compatible with zero for the benchmark energy bin, as shown in the Figure 2(a) for events with $\sec \theta<1.45$. For more inclined events, the parameter A falls rapidly to zero, so that a specific parameterization is needed for this set of events. The result of this new parameterization is shown in the Figure 2(a). As expected, the mean value of the distribution for more inclined events is compatible with zero. 
Merging these datasets ( $\sec \theta<1.45$ and $\sec \theta>1.45$ in Figure 2 ) we see that the new $\left\langle\Delta_{\mathrm{S}}\right\rangle$, Figure 3(a), is consistent with zero. The $\left\langle\Delta_{\mathrm{S}}\right\rangle$ as a function of $\sec \theta$ is shown in the Figure 3(b). In the benchmark region, $\Delta_{\mathrm{S}}$ shows no dependence on the angle.

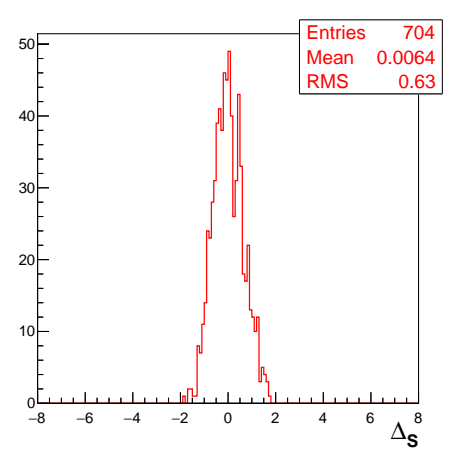

(a) $1.00<\sec \theta<1.45$

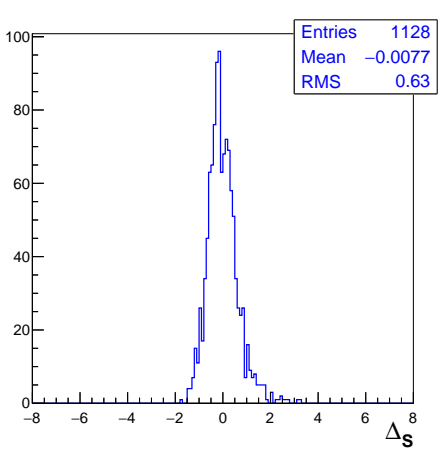

(b) $1.45<\sec \theta<2.00$

Figure 2: $\Delta_{\mathrm{S}}$ values for two zenith angle ranges in the energy bin: $19.1<\log _{10}(\mathrm{E} / \mathrm{eV})<19.2$.

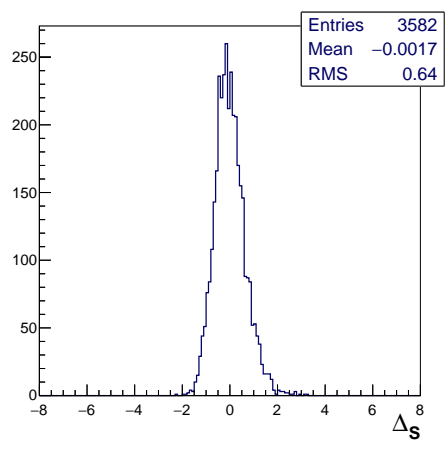

(a) $1.00<\sec \theta<2.00$

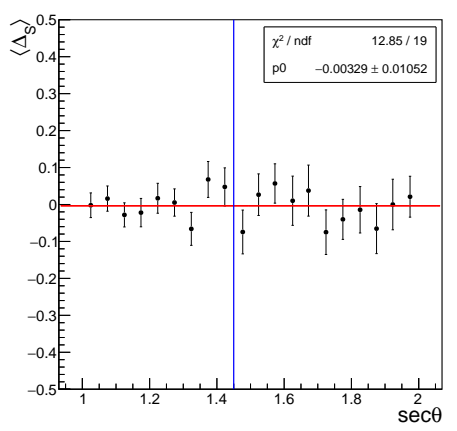

(b)

Figure 3: (a) Distribution of $\Delta_{\mathrm{S}}$ values for the whole angular range considered in this work. (b) Dependence of $\left\langle\Delta_{\mathrm{S}}\right\rangle$ with the zenith angle.

\section{5. $\left\langle\Delta_{\mathbf{S}}\right\rangle$ as function of the energy and the evolution for $\langle\ln \mathbf{A}\rangle$ with energy}

In Figure 4 (Left), the evolution of $\left\langle\Delta_{\mathrm{S}}\right\rangle$ as a function of energy is shown. In Figure 4 (Right), a comparison with hadronic models is made. The measurements are well within the predictions from simulations, and they favour a composition dominated by heavier elements as the energy increases. In Figure 5, good agreement between the new and the previously published data is shown. In total, we have more than doubled the number of events. For $\log _{10}(\mathrm{E} / \mathrm{eV})>19.8$, the data set has been increased by a factor 2.1 because of the larger distance range adopted in the signal selection. Our results confirm, with a bigger statistical sample, that as the energy increases, the composition of the flux is dominated by elements heavier than protons.

In Figure 6, we fit our $\left\langle\Delta_{\mathrm{S}}\right\rangle$ measurements to different functional forms. The fit to a straight line is poor $\left(\chi^{2}=75\right.$ for 13 degrees of freedom). When a broken line is considered, the fit is also 

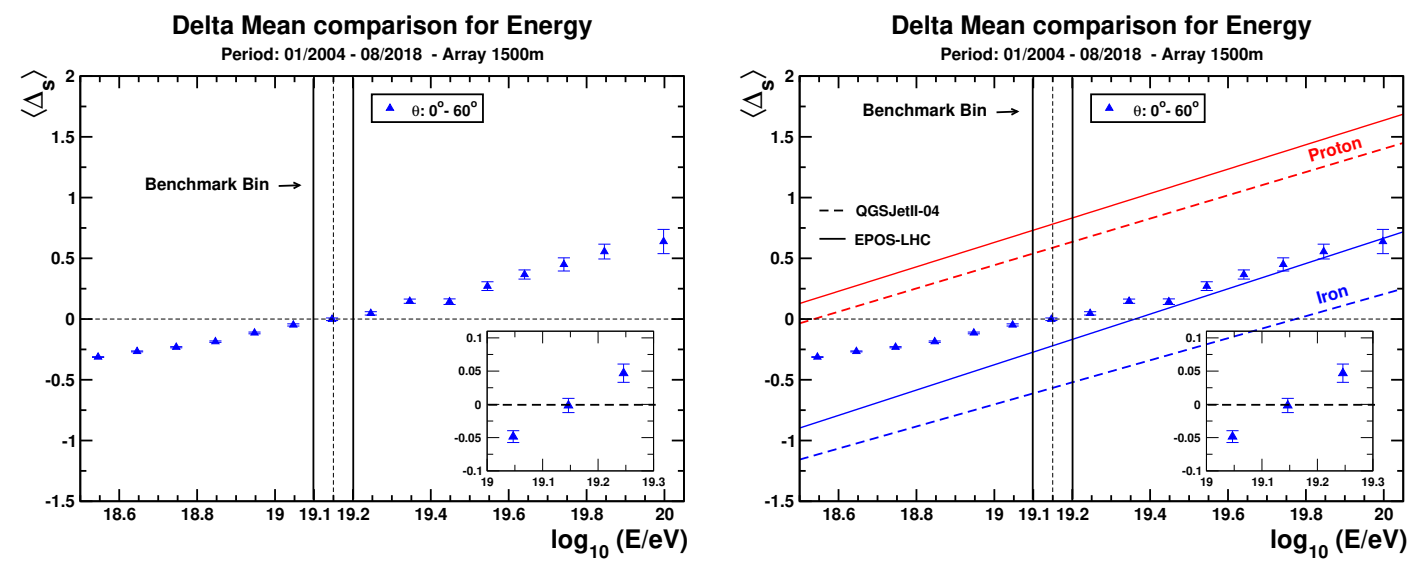

Figure 4: (Left) $\left\langle\Delta_{\mathrm{S}}\right\rangle$ as function of the energy for the new dataset. (Right) Comparison of the evolution of $\left\langle\Delta_{\mathrm{s}}\right\rangle$ as a function of the energy for data and simulations.

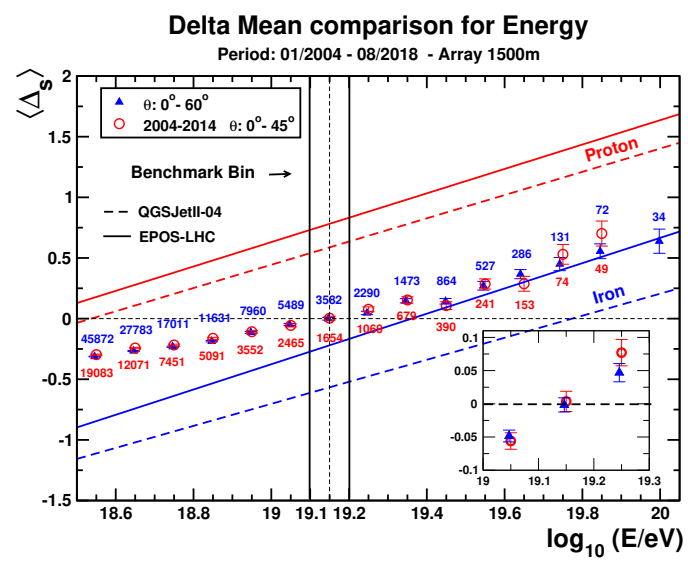

Figure 5: Comparison with previously published results. The numbers indicate the amount of events in each energy bin.

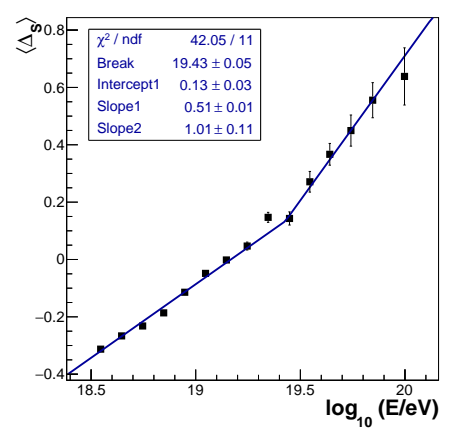

(a) Broken Line.

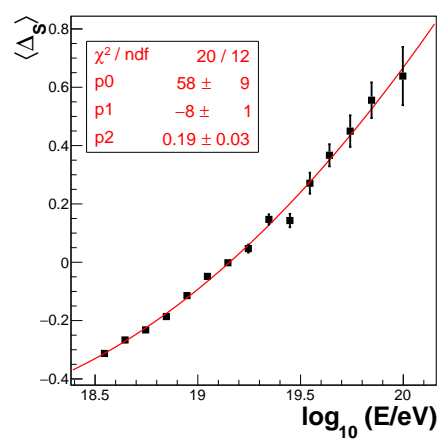

(b) Second degree polynomial.

Figure 6: Fits for $\left\langle\Delta_{\mathrm{S}}\right\rangle$ as a function of the energy. 
poor $\left(\chi^{2} / \mathrm{dof}=42 / 11\right)$. A better fit is obtained $\left(\chi^{2} / \mathrm{dof}=20 / 12\right)$ when our measurements are fit to a second degree polynomial. When compared to model predictions, this result clearly indicates that the rate of change of mass composition varies with energy.

Although it is not possible to compare the fluorescence and surface detector data directly, it is possible to transform the $X_{\max }$ and the $\Delta_{\mathrm{S}}$ values to values of $\langle\ln \mathrm{A}\rangle$. In this work, we used the proton and iron primaries for the hadronic models QGSJetII-04 [6] and EPOS-LHC [7].

Figure 7 shows that the QGSJetII-04 and EPOS-LHC models do not describe data adequately. One of the most prominent reasons for this disagreement comes from the difference observed when comparing the number of predicted muons in models with the measurements made with the Pierre Auger data [8].
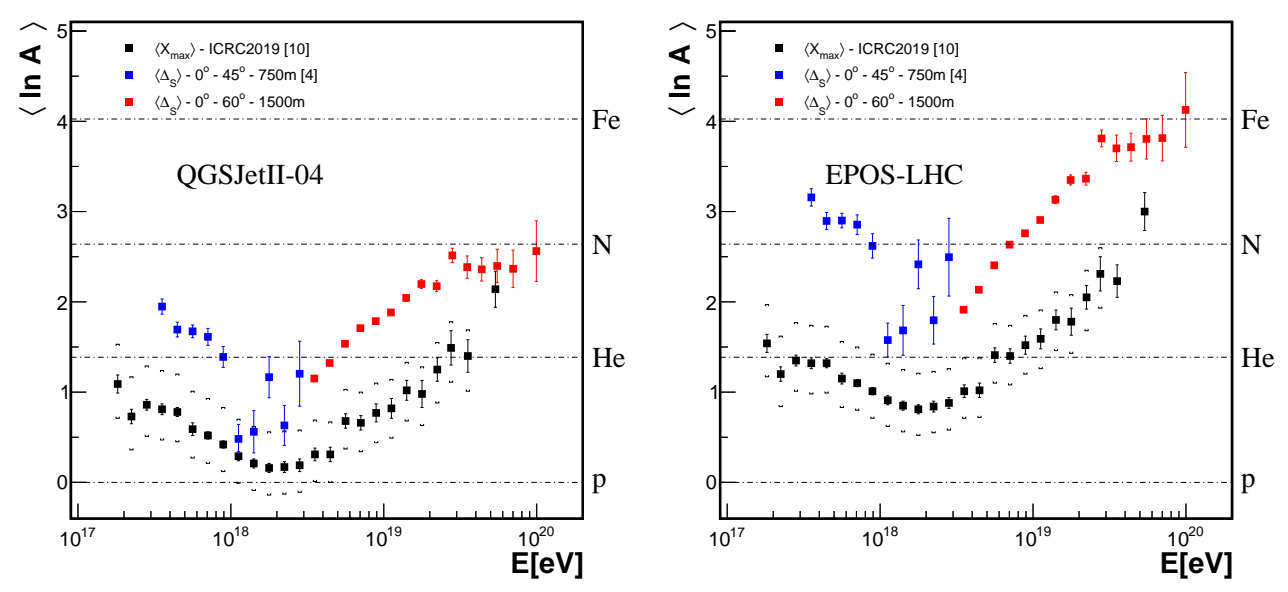

Figure 7: Evolution of the average mass with energy for measurements done with the FD and the SD.

\section{Cross Calibration between $\Delta_{\mathbf{S}}, X_{\max }^{\mathrm{FD}}$ and Energy}

We now address the correlation of $\Delta_{\mathrm{S}}$ with $X_{\max }$ using hybrid events. We would not expect a 1:1 correlation between these parameters because the position of $X_{\max }$ is dominated by the electromagnetic component, whereas $\Delta_{\mathrm{S}}$ is dependent on a muon/electroma- gnetic mix. The values of $\Delta_{\mathrm{S}}$ can be calibrated with $\left\langle X_{\max }^{\mathrm{FD}}\right\rangle$ providing a correlation from which a new parameter $\left\langle X_{\max }^{\text {Delta }}\right\rangle$ can be calculated. These events have been taken from the FD data set discussed in [9] and are shown in Figure 8. There are 2126 events available for calibration. The selected samples of events are unbiased.

Adjusting a linear surface parameterized as

$$
\left\langle X_{\max }^{\text {Delta }}\right\rangle=a+b \cdot\left\langle\Delta_{\mathrm{s}}\right\rangle+c \cdot \log _{10}\left(E_{S D} / \mathrm{eV}\right)
$$

the resulting parameters were used to calculate $\left\langle X_{\max }^{\text {Delta }}\right\rangle$. The values of the parameters $a, b$ and $c$ are listed in Table 1.

The $\left\langle X_{\max }^{\text {Delta }}\right\rangle$ and $\left\langle X_{\max }^{\mathrm{FD}}\right\rangle$ vs energy are shown in Figure 9, together with the number of events (FD and SD) for each energy bin. This analysis confirms with greater precision that, as the energy increases, intermediate/heavy nuclei are preponderant in the flux composition of ultra-high energy cosmic rays. 
Table 1: Values for $a, b$ and $c$ for equation 6.1.

\begin{tabular}{l|c|c|c} 
Array & $a\left(\mathrm{~g} / \mathrm{cm}^{2}\right)$ & $b\left(\mathrm{~g} / \mathrm{cm}^{2}\right)$ & $c\left(\mathrm{~g} / \mathrm{cm}^{2}\right)$ \\
\hline $1500 \mathrm{~m}$ & $733 \pm 38$ & $34 \pm 12$ & $2 \pm 1$
\end{tabular}

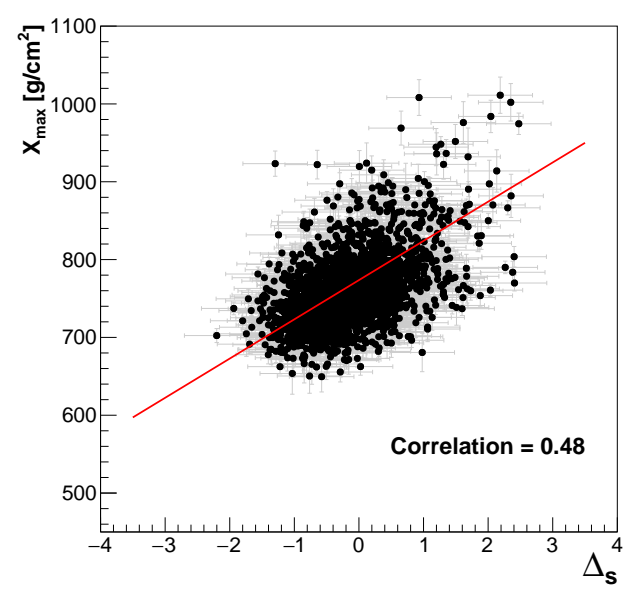

Figure 8: Correlation between $X_{\max }$ and $\Delta_{\mathrm{s}}$ for hybrid events.

\begin{tabular}{|c|c|}
\hline $\log _{10}(\mathrm{E} / \mathrm{eV})$ & $\mathrm{FD}$ \\
\hline $18.5-18.6$ & 1347 \\
$18.6-18.7$ & 1007 \\
$18.7-18.8$ & 707 \\
$18.8-18.9$ & 560 \\
$18.9-19.0$ & 417 \\
$19.0-19.1$ & 321 \\
$19.1-19.2$ & 253 \\
$19.2-19.3$ & 159 \\
$19.3-19.4$ & 122 \\
$19.4-19.5$ & 80 \\
$19.5-19.6$ & 50 \\
$19.6-19.7$ & - \\
$19.7-19.8$ & 35 \\
$19.8-19.9$ & - \\
$>19.9$ & - \\
\hline Total & 5058 \\
\hline
\end{tabular}

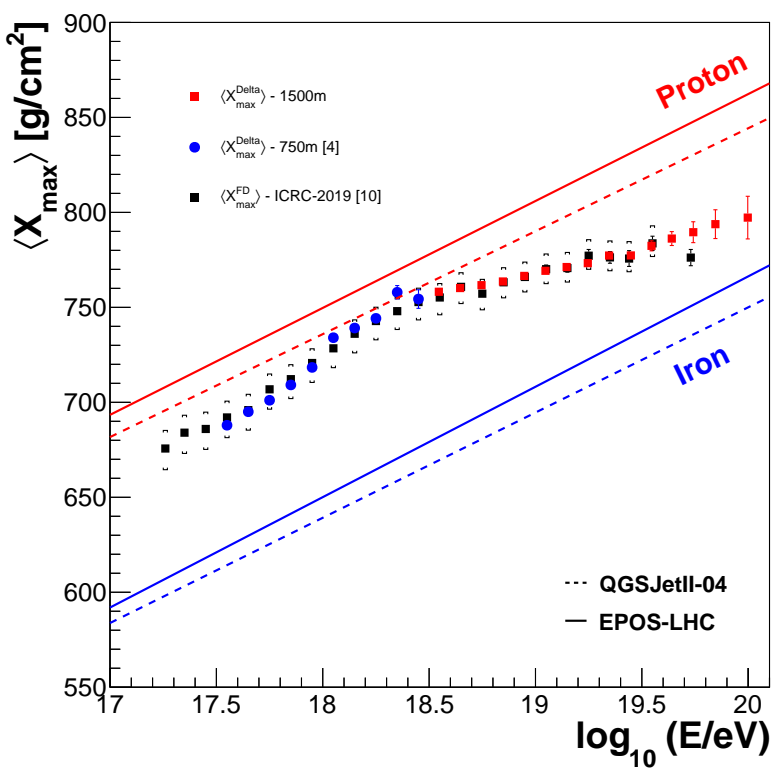

\begin{tabular}{|c|c|}
\hline $\log _{10}(\mathrm{E} / \mathrm{eV})$ & $\mathrm{SD}$ \\
\hline $18.5-18.6$ & 45872 \\
$18.6-18.7$ & 27783 \\
$18.7-18.8$ & 17011 \\
$18.8-18.9$ & 11631 \\
$18.9-19.0$ & 7960 \\
$19.0-19.1$ & 5489 \\
$19.1-19.2$ & 3582 \\
$19.2-19.3$ & 2290 \\
$19.3-19.4$ & 1473 \\
$19.4-19.5$ & 864 \\
$19.5-19.6$ & 527 \\
$19.6-19.7$ & 286 \\
$19.7-19.8$ & 131 \\
$19.8-19.9$ & 72 \\
$>19.9$ & 34 \\
\hline Total & 125005 \\
\hline
\end{tabular}

Figure 9: Evolution of $\left\langle X_{\max }^{\text {Delta }}\right\rangle$ with energy. The SD measurements are compared with the $\left\langle X_{\max }\right\rangle$ measurements done with the FD [10]. The figures in the table correspond to the number of events per energy bin. They are restricted to the energy interval where the Delta method has been updated. 


\section{Conclusions}

The Delta Method uses the information provided by the risetimes of the signals recorded by the WCD of the Surface Detector Array. With it, we can probe the validity of the assumptions contained in state-of-the-art packages that model hadronic interactions at ultra-high energies. Likewise, we can perform mass composition studies with the largest data sample available. This work extends the applicability of the Delta Method up to zenith angles of $60^{\circ}$ and adds three more years of data to previously published results. We confirm, with improved precision, that: i) the rate of change of mass composition varies in the energy range covered by our analysis and, that intermediate/heavy nuclei are predominant as the energy increases; ii) simulations do not reproduce correctly the physics of extensive air showers.

\section{Acknowledgements}

We acknowledge the support from Fundação de Amparo à Pesquisa do Estado de São Paulo (FAPESP) 2016/19764-9; Conselho Nacional de Desenvolvimento Científico e Tecnológico (CNPq) 404993/2016-8 and Financiadora de Inovação e Pesquisa (FINEP). We also acknowledge the National Laboratory for Scientific Computing (LNCC/ MCTI, Brazil) for providing HPC resources of the SDumont and CENAPAD-SP project UNICAMP/ FINEP-MCT, which have contributed to the research results reported within this paper.

\section{References}

[1] Pierre Auger Collaboration, Depth of maximum of air-shower profiles at the Pierre Auger Observatory. I. Measurements at energies above $10^{17.8} \mathrm{eV}$, Phys. Rev. D 90 (2014) 122005.

[2] D. García-Gámez for the Pierre Auger Collaboration, Measurement of atmospheric production depths of muons with the Pierre Auger Observatory, in 32 ${ }^{\text {th }}$ ICRC, (Beijing), August, 2011.

[3] Pierre Auger Collaboration, Azimuthal asymmetry in the risetime of the surface detector signals of the Pierre Auger Observatory, Phys. Rev. D 93 (2016) 072006.

[4] Pierre Auger Collaboration, Inferences on mass composition and tests of hadronic interactions from 0.3 to 100 EeV using the water-Cherenkov detectors of the Pierre Auger Observatory, Phys. Rev. D 96 (2017) 122003.

[5] P. Sánchez-Lucas, Ph.D. thesis: https://hera.ugr.es/tesisugr/26357422.pdf.

[6] S. Ostapchenko, Non-linear screening effects in high energy hadronic interactions, ,Phys. Rev. D 74 (2006) 014026.

[7] K. Werner, F.-M. Liu and T. Pierog, Parton ladder splitting and the rapidity dependence of transverse momentum spectra in deuteron-gold collisions at the BNL relativistic heavy ion collider, Phys. Rev. C 74 (2006) 044902.

[8] Pierre Auger Collaboration, Testing hadronic interactions at ultrahigh energies with air showers measured by the Pierre Auger Observatory, Phys. Rev. Lett. 117 (2016) 192001.

[9] J. A. Bellido for the Pierre Auger Collaboration, Depth of maximum of air-shower profiles at the Pierre Auger Observatory: Measurements above $10^{17.2} \mathrm{eV}$ and composition implications, in $34^{\text {th }}$ ICRC, (Bexco, Busan, Korea), July, 2017.

[10] A. Yushkov for the Pierre Auger Collaboration, Mass composition of cosmic rays with energies above $10^{17.2} \mathrm{eV}$ from the hybrid data of the pierreauger observatory, in PoS(ICRC2019)482. 